\title{
Isomorphic phase transformation in shocked Cerium using molecular dynamics
}

\author{
V. Dupont ${ }^{\mathrm{a}}$, S.-P. Chen ${ }^{\mathrm{b}}$, and T.C. Germann ${ }^{\mathrm{c}}$ \\ Los Alamos National Laboratory, Los Alamos, New Mexico 87545, USA
}

\begin{abstract}
Cerium $(\mathrm{Ce})$ undergoes a significant $(\sim 16 \%)$ volume collapse associated with an isomorphic fcc-fcc phase transformation when subject to compressive loading. We present here a new Embedded Atom Method (EAM) potential for Cerium that models two minima for the two fcc phases. We show results from its use in Molecular Dynamics (MD) simulations of Ce samples subjected to shocks with pressures ranging from 0.5 to $25 \mathrm{GPa}$. A split wave structure is observed, with an elastic precursor followed by a plastic wave. The plastic wave causes the expected fcc-fcc phase transformation. Comparisons to experiments and MD simulations on Cesium (Cs) indicate that three waves could be observed. The construction of the EAM potential may be the source of the difference.
\end{abstract}

\section{Introduction}

Cerium $(\mathrm{Ce})$ has an atypical phase diagram, presenting a large number of unusual structures, a minimum in the melting curve, an isomorphic phase transition between two FCC structures $\alpha$ and $\gamma$, and a critical point around the point $550 \mathrm{~K}$ and $1.9 \mathrm{GPa}$ [1-5]. The $\gamma$ to $\alpha$ phase transition is believed to be caused by the transfer of valence electrons from the $4 f$ state to the $5 d$ state and induces a volume collapse of $\sim 16 \%[1,3]$.

There is an interest in developing multiphase equations of state, and Ce, with its complex phase diagram at low pressure, is an excellent candidate for these studies [6,7]. The isomorphic phase transition has been shown to occur at the same critical pressures under static and dynamic loading [3], so that shock loading is often used to study the phase transformation [3,7].

Shock loading experiments show a two- or three- wave profile depending on the shock pressure $[3,7]$. The three-wave profile consists of an elastic precursor, a plastic wave in the $\gamma$ phase, and the $\gamma$ to $\alpha$ phase transformation. In the experiments leading to a two-wave profile, the elastic precursor is overdriven by the plastic waves.

In this paper, we present an Embedded Atom Method (EAM) potential for Ce and its use in shock loading via MD simulations. We will discuss the choice of potential based on comparisons between our results and experiments, but also based on results from other MD simulations.

\section{Methods}

\subsection{Interatomic potential}

We used a modified Voter-Chen [8] Embedded Atom Method (MVC-EAM) potential to fit the properties of Ce. A detailed description of the MVC-EAM will be given elsewhere [9] and we only provide

\footnotetext{
a e-mail: vdupont@lanl.gov

b e-mail: sc@lanl.gov

c e-mail: tcg@lanl.gov
}

This is an Open Access article distributed under the terms of the Creative Commons Attribution-Noncommercial License 3.0, which permits unrestricted use, distribution, and reproduction in any noncommercial medium, provided the original work is properly cited. 


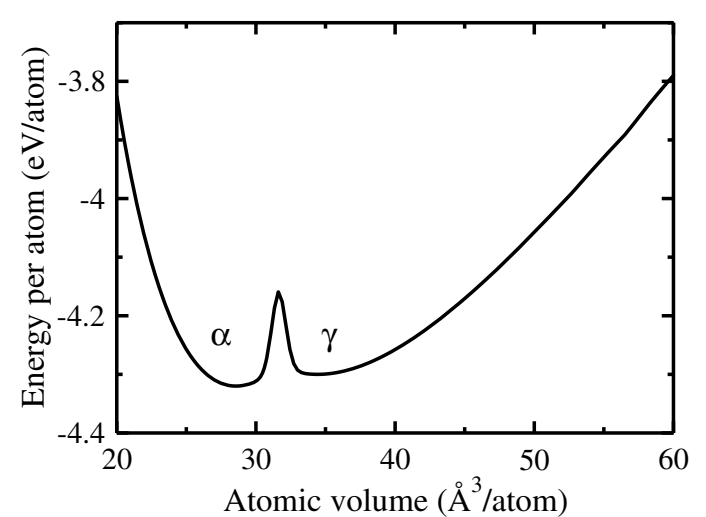

Fig. 1. Cold curve of the fcc phases of Ce using the MVC-EAM potential, showing the two energy wells for each of the fcc phases.

a short summary here. The MVC-EAM differs from the original VC-EAM by replacing the energyvolume curve of one analytical Rose function [10] with a numerical energy-volume curve that is a composite of two Rose functions that describe two phases of $\mathrm{Ce}$ ( $\alpha$ and $\gamma$ phases). We also increased the energy barrier to $0.142 \mathrm{eV} /$ atom with a small Gaussian between the volumes of the $\alpha$ and $\gamma$ phases to ensure their proper stability (Fig. 1). In the MVC-EAM fitting, the pair part is described by a Morse function as for the VC-EAM. The embedding part is extracted in a numerical form to fit in a leastsquares fashion to the properties of cohesive energies, structural parameters, elastic moduli, vacancy energies of different phases. The fitted properties as well as the calculated properties are listed in Table 1.

After the fit, we used the potential to calculate the melting temperature of $\mathrm{Ce}$ and found a value of $1150 \mathrm{~K}$, which is very close to the experimental value of $1071 \mathrm{~K}$ [11], even though the melting properties are not included in the fit. We also found the surface energies of (100), (110) and (111) surfaces to be 896,807 and $845 \mathrm{~mJ} / \mathrm{m}^{2}$ respectively in the $\alpha$ phase, and 557,614 and $551 \mathrm{~mJ} / \mathrm{m}^{2}$ respectively in the $\gamma$ pahse. All of these numbers are in the usual range of other fcc metals. The $\alpha$ (111) stable and unstable stacking fault energies are found to be 19 and $489 \mathrm{~mJ} / \mathrm{m}^{2}$, respectively and -23 and $773 \mathrm{~mJ} / \mathrm{m}^{2}$ for the $\gamma$ phase. The stable stacking fault energy in the $\gamma$ phase is negative because the hcp structure is more stable that the fcc structure. No data was found on these properties so no comparisons were made.

\subsection{Molecular dynamics simulations}

We use the LANL SPaSM (Scalable Parallel Short-range Molecular Dynamics) code, with 40 processors per simulation run. Single crystals of the $\gamma$ phase with two different crystallographic orientations are studied: $\langle 001\rangle$ or $\langle 111\rangle$ along the shock direction. The samples are about $13 \mathrm{~nm} \times 13 \mathrm{~nm} \times 130 \mathrm{~nm}$ and are comprised of around 0.5 million atoms. Periodic boundary conditions are used in the lateral directions. The samples are studied at $10 \mathrm{~K}$ and $300 \mathrm{~K}$. The shock wave is produced by launching the sample into a "momentum mirror" reflecting boundary with a specified velocity $u_{p}[16-21]$.

The local crystallography is analyzed using the angle distribution analysis from [23]. In the following figures, when the angle distribution analysis is used, grey atoms are FCC atoms, blue atoms are $\mathrm{HCP}$ (stacking faults) and red atoms are unknown (surfaces and other defects). The local orientation around an atom is determined using the method described in [21].

\section{Results and discussion}

A typical velocity profile is shown in Fig. 2. Two shock waves can be observed in this example. The first wave is an elastic precursor, and in order to determine the nature of the second wave, a 
New Models and Hydrocodes for Shock Wave Processes in Condensed Matter

Table 1. Fitted and calculated properties of MVC-EAM for Ce.

\begin{tabular}{lccc}
\hline Property & Exptl. / Calc. & Fit / Calc. & Deviation $(\%)$ \\
\hline$\alpha$-fcc, $a_{0}(\AA)$ & $4.850^{\mathrm{a}}$ & 4.8526 & - \\
$E_{c}(\mathrm{eV} /$ atom $)$ & $4.32^{\mathrm{b}}$ & 4.32 & - \\
$B(\mathrm{GPa})$ & $37.0^{\mathrm{c}}$ & 37.053 & - \\
$C_{11}(\mathrm{GPa})$ & $52.9^{\mathrm{c}}$ & 56.828 & +7.4 \\
$C_{12}(\mathrm{GPa})$ & $29.1^{\mathrm{c}}$ & 27.165 & -6.6 \\
$C_{44}(\mathrm{GPa})$ & $44.6^{\mathrm{c}}$ & 41.371 & -7.2 \\
$E_{v}^{f}(\mathrm{eV})$ & $1.71^{\mathrm{d}}$ & 1.4795 & -13.5 \\
& & & \\
$\gamma$-fcc, $a_{0}(\AA)$ & $5.161^{\mathrm{a}}$ & 5.1637 & - \\
$E_{c}(\mathrm{eV} /$ atom $)$ & $4.3^{\mathrm{e}}$ & 4.3 & - \\
$B(\mathrm{GPa})$ & $18.18^{\mathrm{f}}$ & 18.265 & +0.5 \\
$C_{11}(\mathrm{GPa})$ & $26.01^{\mathrm{f}}$ & 24.145 & -7.2 \\
$C_{12}(\mathrm{GPa})$ & $14.26^{\mathrm{f}}$ & 15.326 & +7.5 \\
$C_{44}(\mathrm{GPa})$ & $17.3^{\mathrm{f}}$ & 18.188 & +5.1 \\
$E_{v}^{f}(\mathrm{eV})$ & $1.5^{\mathrm{g}}$ & 1.1888 & -21.7 \\
& & & \\
$\mathrm{bcc}, a_{0}(\AA)$ & $4.12^{\mathrm{a}}$ & 4.123 & +0.1 \\
$E_{c}(\mathrm{eV} /$ atom $)$ & $4.09^{\mathrm{h}}$ & 4.2944 & +5.0 \\
& & & \\
hcp, $a_{0}(\AA)$ & $4.667^{\mathrm{d}}$ & 4.372 & -6.3 \\
$E_{c}(\mathrm{eV} /$ atom $)$ & $4.24^{\mathrm{i}}$ & 4.1655 & -1.8 \\
\hline
\end{tabular}

${ }^{a}[11]$

$\mathrm{b}[12]$

${ }^{c}[13]$

${ }^{\mathrm{d}}$ Present work using VASP by S.P. Chen

e $[5,14]$

${ }^{\mathrm{f}}[15]$

${ }^{\mathrm{g}}$ Estimated value

${ }^{\text {h }}$ From energy difference of $\alpha$-fcc and bcc using VASP by S.P. Chen

${ }^{\text {i }}$ From energy difference of $\alpha$-fcc and hcp using VASP by S.P. Chen

shocked sample is represented with its angle distribution analysis, as well as the radial distribution function of the sample before and after the shocks (Fig. 3). The angle distribution analysis shows that stacking faults have been left behind partial dislocations after the second shock wave. Plasticity is thus sustained after the second shock, and at the same time, the lattice parameter after the second shock has decreased below the value of the lattice parameter of the $\alpha$ phase $(4.85 \AA)$. The phase transformation thus occurs before any plasticity has been sustained in the original $\gamma$ phase. This can be explained by looking at the energies required to initiate the phase transformation and the energy required to nucleate a stacking fault in the $\gamma$ phase (unstable stacking fault energy). The energy barrier between the two phases was chosen to be $0.142 \mathrm{eV} /$ atom. In comparison, the unstable stacking fault energy in the $\gamma$ phase is $773 \mathrm{~mJ} / \mathrm{m}^{2}$, which corresponds to $0.245 \mathrm{eV} /$ atom for the two layers of atoms on each side of the staking fault. It is thus understandable that the phase transformation will occur before any plasticity can be sustained in the $\gamma$ phase.

We show in Fig. 4 the $U_{s}-u_{p}$ Hugoniots for the two loading directions and the two temperatures, along with a fit to experiments [22]. We first observe that our simulations reasonably agree with the experimental data, especially at low $u_{p}$. The orientation of the sample and the initial temperature have very little effect on the simulations, and especially on the phase transformation. The time and length scale of our simulations vary greatly with those of the experiments and might account for the differences. In addition, our samples are in a perfect lattice at the beginning of the simulation and are single crystalline. 


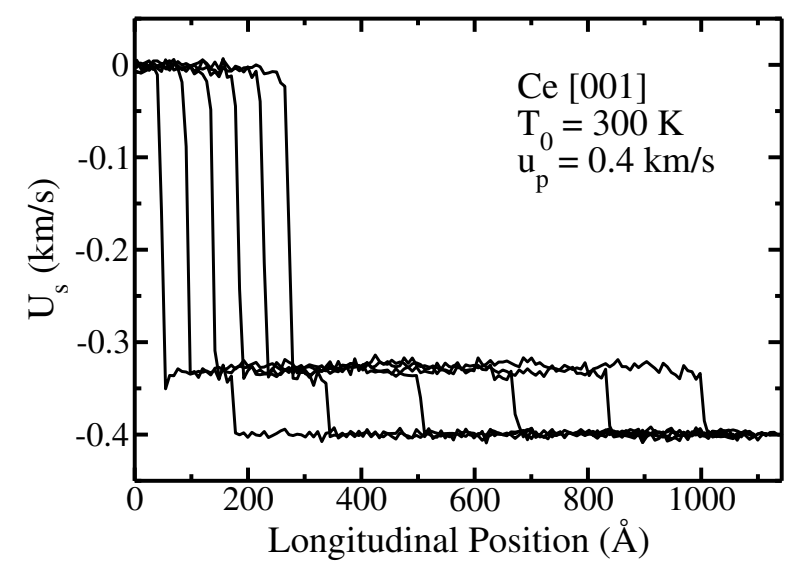

Fig. 2. Velocity profiles, spaced 4 ps apart, of Ce shocked in the [001] direction with a particle velocity of $0.4 \mathrm{~km} / \mathrm{s}$. These velocity profiles show a 2-wave structure: an elastic precursor and a phase transformation accompanied by plasticity.
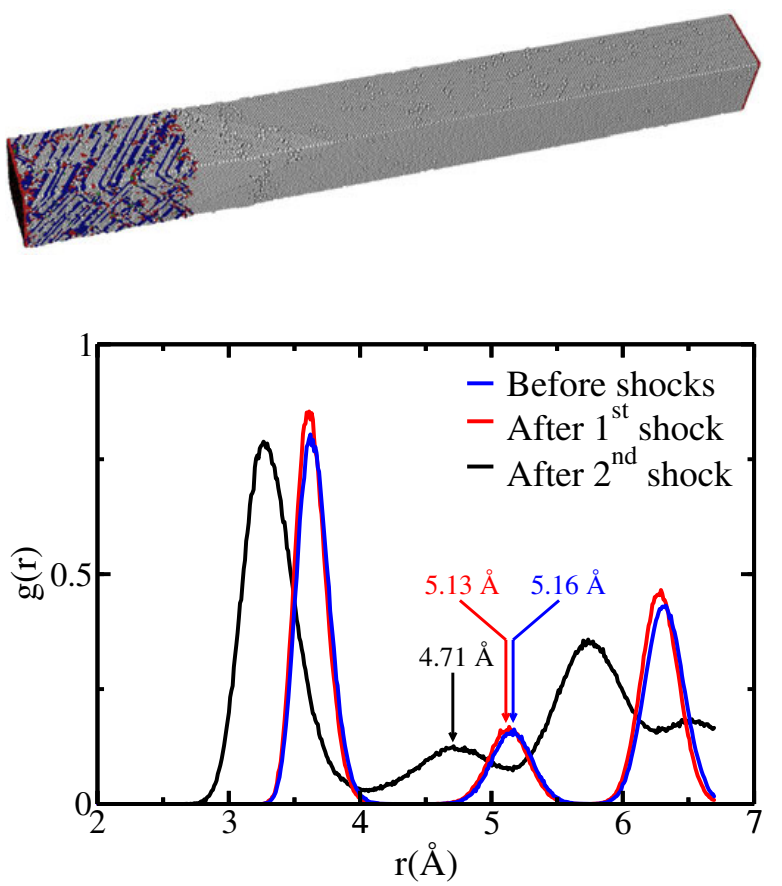

Fig. 3. (Top) Angle distribution analysis representation on a sample shocked in the [001] direction at $300 \mathrm{~K}$ with $u_{p}=0.4 \mathrm{~km} / \mathrm{s}$ (Bottom) Rdf on slices after each shock. These figures show that plasticity occurs after the phase transformation.

In simulations of shock in Cs, it was shown that depending on the initial orientation of the sample, the phase transformation would lead to a re-orientation of the lattice [21]. Orientation analysis on our simulations showed that no re-orientation occurred.

\section{Conclusions}

We used a recent Modified Voter-Chen Embedded Atom Method potential for Ce under shock. This potential is characterized by two energy wells in the FCC phase, representing the two phases of Ce. We 

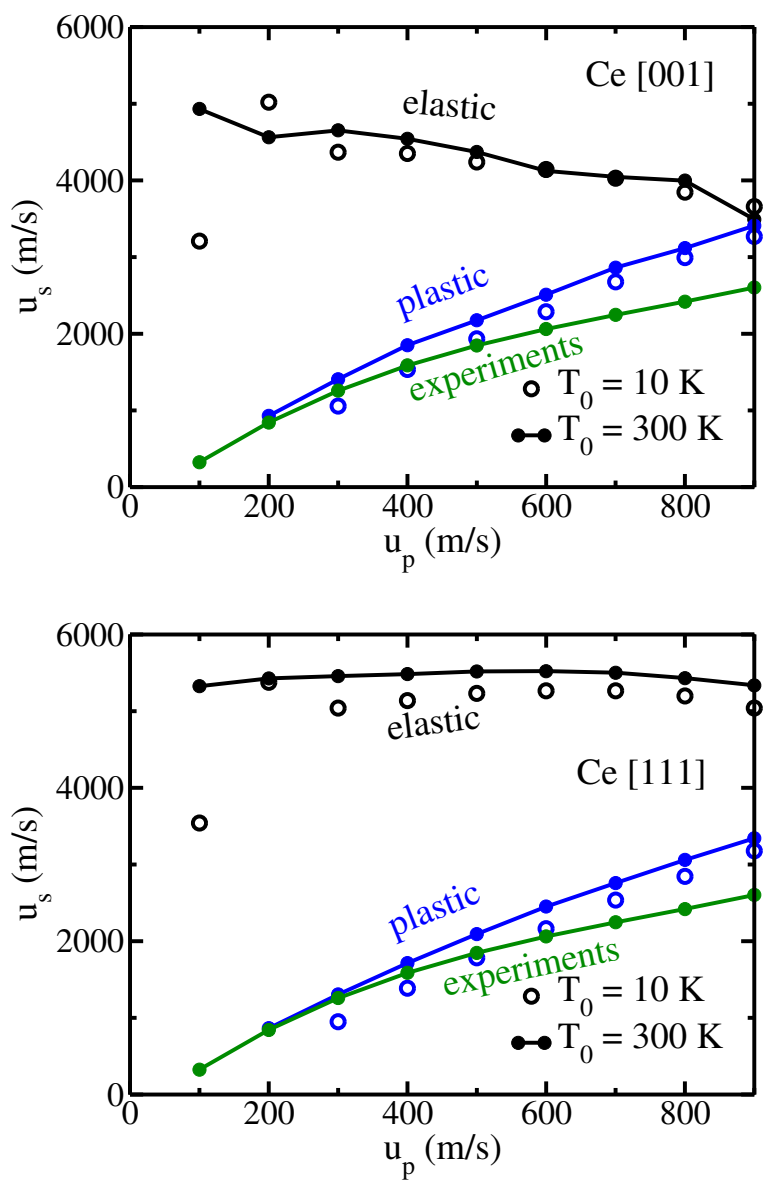

Fig. 4. Us-Up Hugoniots of Ce for two different orientations and initial temperatures. The curves labeled "experiments" correspond to a fit to experiments from [22].

have shown that the use of such potential for shock simulations is possible. However, we found that the current potential does not show a 3-wave structure as observed experimentally [3] or in Cs simulations [21]. Our $U_{s}-U_{p}$ Hugoniots show that temperature and orientation of the lattice have little effect on the phase transformation. Further experimental and theoretical work is needed to explain the behavior of Ce under shock loading. Two options for the theoretical study are to either increase the energy barrier between the two stable phases, or use a different type of potential, such as the potential developed by Ackland et al [24] and used in the Cs study [21].

\section{References}

1. A.W. Lawson, T.Y. Tang, Phys. Rev. 76, 301 (1949)

2. Y. Zhao, W.B. Holzapfel, J. Alloys Compd. 246, 216 (1997)

3. M.N. Pavlovskii, V.V. Komissarov, A.R. Kutsar, Combustion, Explosion, and Shock Wave 35, 88 (1999)

4. N. Richard, S. Bernard, J. Alloys Compd. 323-324, 628 (2001)

5. A. Schiwek, F. Porsch, W.B. Holzapfel, High Press. Res. 22, 407 (2002)

6. F.J. Cherne, B.J. Jensen, V.M. Elkin, Shock Compression of Condensed Matter (edited by M.L. Elert, W.T. Buttler, M.D. Furnish, W.W. Anderson and W.G. Proud, American Institute of Physics, 2009) 1161 
7. B.J. Jensen, Shock Compression of Condensed Matter (edited by M.L. Elert, W.T. Buttler, M.D. Furnish, W.W. Anderson and W.G. Proud, American Institute of Physics, 2009) 1165

8. A.F. Voter, S.P. Chen, Mat. Res. Soc. Symp. Proc. 82, 175 (1987)

9. S.P. Chen, T.J. Gammel, J.D. Kress, V. Dupont, T.C. German (to be published)

10. J.H. Rose, J.R. Smith, F. Guinea, J. Ferrante, Phys. Rev. B 29, 2963 (1984)

11. T.B. Massalski, Ed., Binary Alloy Phase Diagrams (American Society for Metals, Metals Park, OH 44073, 1986), vol. 2, p. 2175

12. C. Kittel, Introduction to Solid State Physics (John Wiley, 7th Ed., 1996) p. 57

13. P. Soderlind, O. Eriksson, J.M. Wills, A.M. Boring, Phys. Rev. B 48, 9306 (1993)

14. R.I. Beecroft, C.A. Swenson, J. Phys. Chem. Solids 15, 234 (1960)

15. J.D. Greiner, O.D. McMasters, J.F. Smith, Scr. Met. 14, 989 (1980)

16. B.L. Holian, P.S. Lomdahl, Science 280, 2085 (1998)

17. T.C. Germann, B.L. Holian, P.S. Lomdahl, R. Ravelo, Phys. Rev. Lett. 84, 5351 (2000)

18. T.C. Germann, D. Tanguy, B.L. Holian, P.S. Lomdahl, M. Mareschal, R. Ravelo, Metal. and Mater. Trans. A 35, 2609 (2004)

19. K. Kadau, T.C. Germann, P.S. Lomdahl, B.L. Holian, Science 296, 1681 (2002)

20. K. Kadau, T.C. Germann, P.S. Lomdahl, B.L. Holian, Phys. Rev. B 72, 064120 (2005)

21. T.C. Germann, K. Kadau, Shock Compression of Condensed Matter (edited by M.L. Elert, W.T. Buttler, M.D. Furnish, W.W. Anderson and W.G. Proud, American Institute of Physics, 2009) 761

22. B.J. Jensen, F.J. Cherne, J.C. Cooley, M.V. Zhernokletov, A.E. Kovalev, Phys. Rev. B 81, 214109 (2010)

23. G.J. Ackland, A.P. Jones, Phys. Rev. B 73, 054104 (2006)

24. G.J. Ackland, S.K. Reed, Phys. Rev. B 67, 174108 (2003) 